\title{
In situ TEM observations of Oxygen Surface Dynamics in $\mathrm{CeO}_{2} \mathrm{Cubes}$
}

\author{
Ethan L. Lawrence ${ }^{1}$, Shery L. Y. Chang ${ }^{2}$, \& Peter A. Crozier ${ }^{1}$ \\ 1. School for the Engineering of Matter, Transport and Energy, Arizona State University, Tempe Arizona \\ 85287-6106. \\ 2. LeRoy Eyring Center for Solid State Science, Arizona State University, Tempe, Arizona 85287-1704, \\ USA.
}

The fundamental steps and active sites in the oxygen reduction reaction (ORR) on reducible oxide surfaces is an outstanding fundamental problem in heterogeneous catalysis. For many applications, such as fuel cells, the oxygen exchange reaction is often the rate limiting step and it is critical to develop an atomic level fundamental understanding of the reaction pathways. Reducible oxides such as $\mathrm{CeO}_{2}$ (ceria) are ideal model systems to investigate the oxygen exchange reaction. Under reducing conditions, ceria releases oxygen through the formation of an oxygen vacancy coupled with a transition from $\mathrm{Ce}^{4+}$ to $\mathrm{Ce}^{3+}[1]$. The ORR takes place when reduced ceria is exposed to an oxidizing environment. High-resolution transmission electron microscopy (HRTEM) has been used to study Ce atomic movement on ceria nanoparticle surfaces [2]. Recently, atomic surface structures of $\mathrm{CeO}_{2}$ cubes with $\mathrm{O}$ atoms clearly visible were imaged using aberration-corrected HRTEM [3]. Although performing HRTEM imaging of $\mathrm{CeO}_{2}$ under high vacuum conditions at room temperature provides valuable insights, the catalytically important applications of ceria occur at elevated temperatures in gaseous environments. In situ environmental transmission electron microscopy (ETEM) can be used to observe dynamical processes that occur on ceria surfaces in reducing/oxidizing environments at elevated temperatures. Observing oxygen surface dynamics on $\mathrm{CeO}_{2}$ will provide information on the fundamentals of the oxygen exchange reaction that is crucial for catalysis. Here we show dynamic changes on ceria related to oxygen exchange at the surface.

An aberration-corrected FEI Titan ETEM equipped with a Gatan K2 IS direct detection camera (with high detection quantum efficiency and capable of up to $1600 \mathrm{fps}$ ) was used to image $\mathrm{CeO}_{2}$ nanocubes. The image corrector of the microscope was tuned to an optimum negative $\mathrm{C}_{\mathrm{s}}$ condition to enhance contrast from weakly scattering oxygen atomic columns. JEMS software was used for simulations to determine ideal experimental variables (thickness, defocus, zone axis) and to aid in experimental image intensity interpretation [4].

Figure 1a) shows a HRTEM image of a $\mathrm{CeO}_{2}$ cube in (100) projection. A thin region is exposed at the edge of the cube and is magnified in Figure $1 \mathrm{~b}$ ). $\mathrm{Ce}$ and $\mathrm{O}$ atomic columns are both visible at the surface and in this case the surface termination contrast in the image is dominated by the cations although fainter contrast from an incomplete oxygen layer is also visible. A simulated image and structural model are overlaid onto the experimental image showing good agreement. Oxygen removal from the surface is likely to give rise to cation motion. In our initial experiments, we used the electron beam to remove oxygen and trigger cation motion. Atomic motion at surface step edge sites was observed as shown in Figure 2. At time $=0$ seconds, Figure 2a) highlights $\mathrm{Ce}$ and $\mathrm{O}$ atomic columns at a step edge. Figure $2 \mathrm{~b}$ ) was taken at time $=0.3$ seconds and showed that the $\mathrm{Ce}$ and $\mathrm{O}$ atomic columns highlighted in a) had moved. Furthermore, the Gatan K2 IS camera was also operated at 800 fps to capture temporally resolved atomic motion of the surface at room temperature. Low electron dose in situ experiments in gaseous environments and elevated temperatures will be performed to observe oxygen surface dynamics on $\mathrm{CeO}_{2}$ cubes and results will be presented [5]. 
References:

[1] Skorodumova, N.V., et al, Physical Review Letters, 89 (2002), p. 166601.

[2] Bhatta, U.M., et al, ACS Nano, 6 (2012), p. 421-430.

[3] Lin, Y., et al, Nano Letters, 14 (2014), p. 191-196.

[4] Stadelmann, P. JEMS, Electron Microscopy Software, Java Version 4.4831U2016; CIME-EPFL:

Laussane, Switzerland.

[5] We gratefully acknowledge support of NSF grant DMR-1308085, the use of ASU's John M. Cowley Center for High Resolution Electron Microscopy and use of the K2 IS camera courtesy of Gatan.
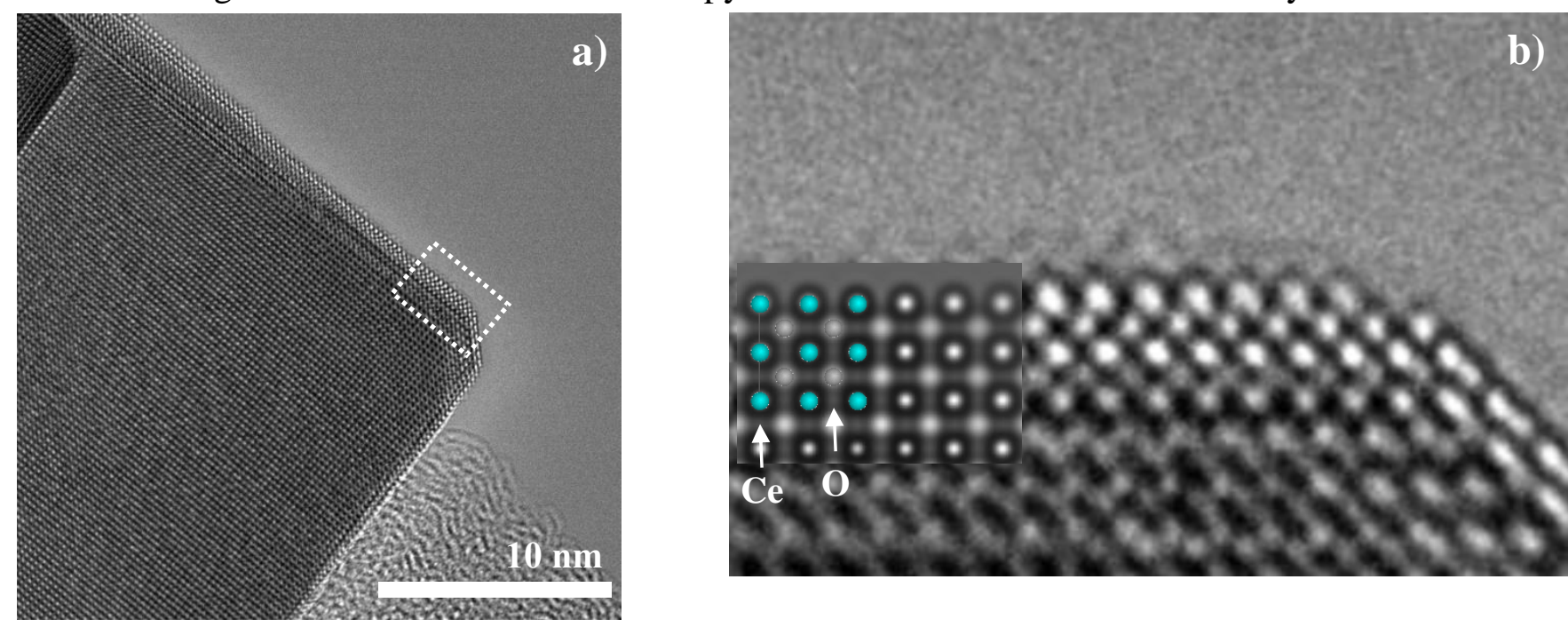

Figure 1. a) HRTEM image of a $\mathrm{CeO}_{2}$ cube in (100) projection. A thin region is exposed at the edge of the cube. b) A magnified view of the region highlighted in the dotted box in a). An image simulated with JEMS software and an atomic model is overlaid on top of the experimental image to show agreement between experiment and theory.
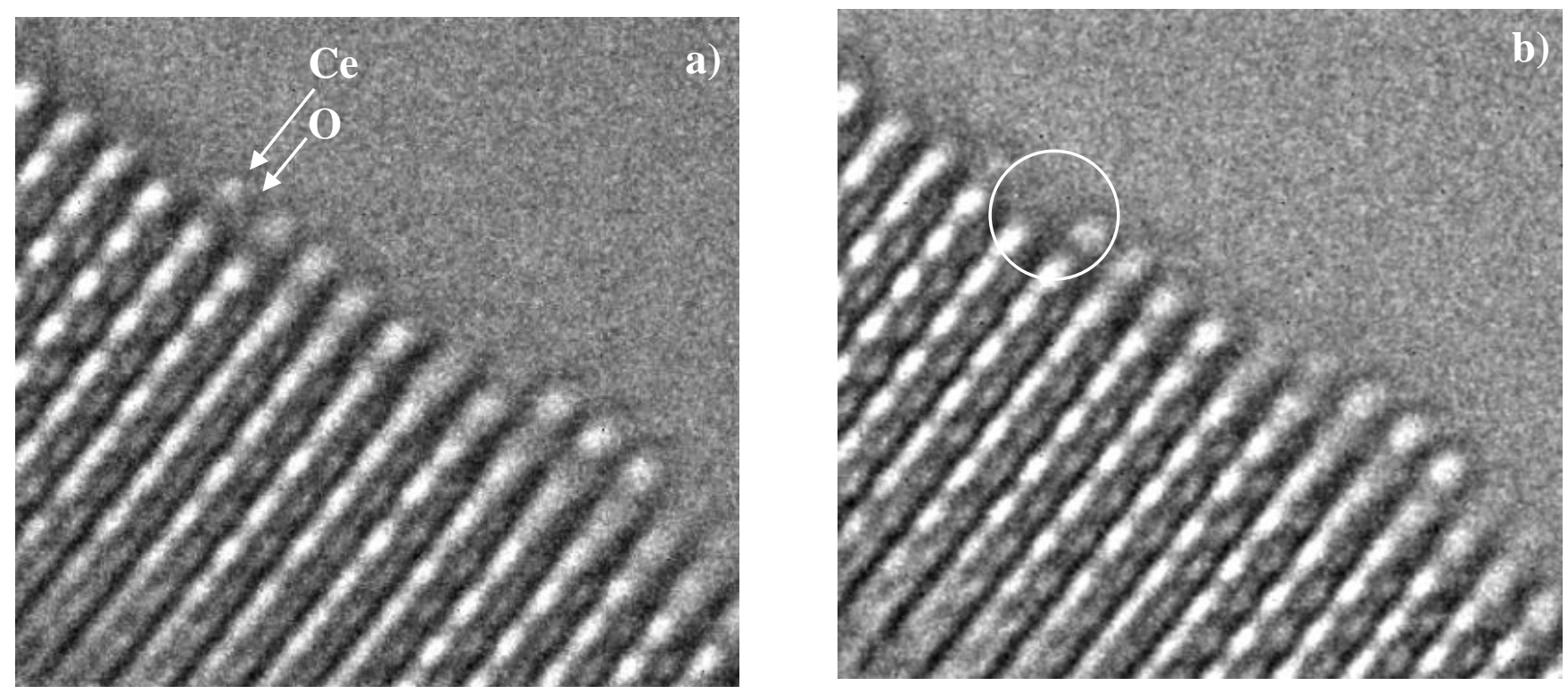

Figure 2. HRTEM images of the surface of a $\mathrm{CeO}_{2}$ cube in (100) projection. a) time $=0$ seconds. b) time $=0.3$ seconds. The surface $\mathrm{Ce}$ and $\mathrm{O}$ atomic columns highlighted in a) have moved and are not visible in $b)$. 